\title{
"Clinical and Microbiological Evaluation of Bloodstream Infections in Patients Undergoing Maintenance Hemodialysis: A Study in Bangabandhu Sheikh Mujib Medical University, Dhaka, Bangladesh"
}

\author{
Md. Siddiqur Rahman ${ }^{1 *}$, Md. Shahidul Islam (Selim) ${ }^{2}$, Md. Ruhul Amin ${ }^{3}$, Md. Ajfar Sazid Khan ${ }^{4}$, Salina Akter \\ ${ }^{1}$ Assistant professor, Department of Nephrology, Rajshahi Medical College Hospital, Rajshahi, Bangladesh \\ ${ }^{2}$ Professor \& Chairman, Department of Nephrology, Bangabandhu Sheikh Mujib Medical University, Dhaka, Bangladesh \\ ${ }^{3}$ Medical Officer, Dialysis Unit, Rajshahi Medical College Hospital, Rajshahi, Bangladesh \\ ${ }^{4}$ Classified Specialist in Medicine \& Nephrologist, Combined Military Hospital, Dhaka, Bangladesh \\ ${ }^{5}$ Specialist, Nephrology, United Hospital Limited, Bangladesh
}

DOI: $10.36348 /$ sjmps.2020.v06i01.002

| Received: 14.12.2019 | Accepted: 23.12.2019 | Published: 06.01.2020

*Corresponding author: Md. Siddiqur Rahman

\section{Abstract}

Introduction: Chronic kidney disease (CKD) is one of the most common non communicable diseases throughout the world. According to the data of Bangladesh Renal Registry almost twenty millions of Bangladeshi adults are suffering from various stages of CKD. Many of these CKD patients ultimately developed ESRD when life is not sustainable unless renal replacement therapy is initiated. Objective: The purpose of the present study was to estimate the rates of infections and clinical and microbiological evaluation of bloodstream infections in patients undergoing maintenance hemodialysis. Methods: This cross sectional study was conducted from January 2014 to December 2015 for a period of 2(two) years in the Department of Nephrology at Bangabandhu Sheikh Mujib Medical University, Dhaka. All adult patients underwent hemodialysis of both sexes fulfilling the inclusion and exclusion criteria were included in the study. Then each patient was evaluated during each hemodialysis session for the (1) presence of bloodstream infection (BSI) using CDC (Centre for disease control) case definitions by blood cultures (Two samples were sent for cultures one from peripheral veins and another from vascular access either from fistula or from central venous catheter) and (2) clinical features. Result: The mean age of the patients were 38.26 (15.26) years. In this present study male $(71.7 \%)$ are predominant than female (28.3\%). Maximum (51.7\%) patients were under weight. In this study 50 (83.3\%) patients had anemia. Mean (SD) WBC, $\mathrm{Hb}$, S. uric acid, FPG, Plasma glucose $2 \mathrm{~h} \mathrm{ABF}, \mathrm{HbA1C}$, S. total protein and S. albumin were $14415(4000) \mathrm{count} / \mathrm{cmm}$, 9.61 (1.62) g/dl, 417.67 (100.87) $\mu \mathrm{mol} / \mathrm{l}, 5.37$ (1.18) mmol/l, 7.27 (2.18) mmol/l, $6.42 \%$ (0.68), 62.71 (5.82) g/l and 28.97 (4.99) g/l respectively. Out of $46 \mathrm{AV}$ fistula cases, culture was positive in vein $8(13.3 \%)$ cases and in fistula 5 $(10.8 \%)$ cases. Out of 11 permanent CV catheter cases, culture was positive in vein $6(54.5 \%)$ cases and in catheter 7 $(63.3 \%)$ cases. Out of 3 catheter tip cases, culture was positive in catheter tip $2(66.7 \%)$ cases. Among 28 bacteremic episodes Pseudomonas aeruginosa were 53.57\%, Acinetobacter spp. were $35.71 \%$ and Klebsiella spp. were $10.71 \%$. Blood stream infection rate due to hemodialysis in fistula was $0.24 / 1000$ patient-days, in catheter was $3.05 / 1000$ patientdays and total was 1.19/1000 patient-days. Acinetobacter spp (6.7\%) and Pseudomonas aeruginosa (16.7\%) were found in peripheral vein. Acinetobacter spp in 3(6.5\%) and Pseudomonas aeruginosa were present in $2(4.3 \%)$ cases in AV fistula. Klebsiella spp. were present in $3(21.4 \%)$ cases, Acinetobacter spp were in $3(21.4 \%)$ cases and Pseudomonas aeruginosa were in $3(21.4 \%)$ cases in Central venous catheter. Conclusion: The risk of BSI in patients undergoing hemodialysis is related to the vascular access (tunneled central venous catheter), low haemoglobin, underweight, low serum total protein and low serum albumin.

Keywords: Mevaluation, Bloodstream infections, Plasma glucose $2 \mathrm{~h}$ ABF, BSI, CDC.

Copyright @ 2020: This is an open-access article distributed under the terms of the Creative Commons Attribution license which permits unrestricted use, distribution, and reproduction in any medium for non-commercial use (NonCommercial, or CC-BY-NC) provided the original author and source are credited.

\section{INTRODUCTION}

Chronic kidney disease (CKD) is one of the most common non communicable diseases throughout the world. According to the data of Bangladesh Renal Registry almost twenty millions of Bangladeshi adults are suffering from various stages of CKD. Many of these CKD patients ultimately developed ESRD when life is not sustainable unless renal replacement therapy is initiated. Incidence rate (number of patients/million population/year) and prevalence (number of patient/million) of ESRD in different countries (2010) : 
In USA : incidence rate 348 and prevalence rate 1752 , In Japan incidence rate 288 and prevalence rate 2260 and in Bangladesh incidence rate 20 and prevalence rate 158 [1].Options for renal replacement therapy in Bangladesh are: 1) Pre-emptive transplantation, 2) Transplantation after starting hemodialysis, 3) In-center hemodialysis 4) Intermittent peritoneal dialysis and 5) Continuous ambulatory peritoneal dialysis (CAPD). Renal transplantation is the best form of renal replacement therapy though it has some disadvantages like insufficient suitable donor, need for compliance with immunosuppression drugs. Continuous ambulatory peritoneal dialysis (CAPD): it preserves residual renal function, have independence and relatively simple but it is associated with high rate peritonitis. Most of the countries of the world and even in Bangladesh hemodialysis is the mostly used renal replacement therapy. A small amount of glucose is also commonly used. Note that this is a different process to the related technique of hemofiltration [2]. Bleeding may also occur; again the risk varies depending on the type of access used. Infections can be minimized by strictly adhering to infection control best practices [3]. Ideally hemodialysis access would be established in advance of starting dialysis. Functional vascular access is needed for all extracorporeal dialytic therapies and remains the life line for patients with ESRD who need chronic intermittent hemodialysis therapy. Ideally a first access should be an aretiovenous fistula placed at the wrist though upper arm access site also used. The next is central venous catheter (CVC) either permanent tunneled CVC or temporary untunneled CVC. In Bangladesh, vascular access for maintenance hemodialysis patients is AV fistula and untunneled CVC. Some hemodialysis centers in Bangladesh are using tunneled CVC, but the rates are still not high. AV grafts rarely used in our country. Data from the Dialysis Outcomes and Practices Patterns Study (DOPPS) shows that in the United States $25 \%$ of dialysis patients are dialyzed with catheters; in other countries the use of catheters is even more common (Belgium, 41\%; UK, $28 \%$ ). Over $70 \%$ of patients with initiating chronic hemodialysis in the United States have a tunneled CVC as their first blood access device [4].Though the native AV fistulas are still the best suited accesses for hemodialysis, this option is impractical in many situations, so that several justifiable reasons exist for the use of CVC; these include the catheter as a bridge angioaccess device, while the patient is awaiting living-related kidney donor transplantation or maturation of an autologus fistula or graft, as the permanent vascular access for the patients unsuitable vascular anatomy who have exhausted all other options [5]. There are some advantages of tunneled CVC over untunneled CVCs: a tunneled CVC is associated with a reduced morbidity, mortality, infection rate as well as better and constant performance compared with untunneled catheter [6] observed that typical infections rate are 3 episodes of infection/ 1000 tunneled catheter days in tunneled catheter and infection rates are high with nontunneled catheter. Vascular access is an established risk factor for sepsis in patients on renal replacement therapy, and different types of haemodialysis vascular access are associated with differing rates of bacteraemia. Patients dialysing through a central venous catheter are most at risk, with an associated bacteraemia incidence of 2-6 cases per 1000 catheter days. Within this group, those dialysing through a non-tunnelled central venous catheter have comparatively higher rates of bacteraemia than those using tunnelled central venous catheters [7]. Optimal survival and the quality of life of patients with end stage renal disease on long term hemodialysis is largely dependent upon the adequacy of dialysis through an appropriately placed and properly functioning permanent vascular access with minimal mechanical complications and infection rates. Vascular access related bloodstream infections and related complications requiring hospitalization, account for nearly one third of the cost of ESRD management with reported mortality rates of $12-25.9 \%$ [8]. Yet, despite these risks and international and national guidelines that recommend fistulas as the preferred form of dialysis access, the proportional use of tunneled catheters for dialysis access has steadily increased in many countries [32].Vascular access related bloodstream infections are a leading cause of hospitalization in patients treated with chronic hemodialysis. In addition, a catheter is used for the initial dialysis session in approximately 80 percent of incident hemodialysis patients [33].The majority of bacteremia in hemodialysis patients are caused by infection of vascular access catheters. In addition, catheter-dependent hemodialysis patients have a two- to threefold higher risk of infection-related hospitalization and infection-related death as compared with patients undergoing dialysis via a fistula or graft [34'35]. Female gender, presence of diabetes mellitus, presence of central venous catheter rather than $\mathrm{A} / \mathrm{V}$ fistula, low serum total protein, low serum albumin, high white cell count and low hemoglobin levels are associated with bloodstream infection [30]. Catheter can sometimes became colonized from more remote sites during bacteremia [36]. Bloodstream infection (BSI) is the leading cause of hospitalization and the second most common cause of death among patients receiving hemodialysis [29]. There may also be symptoms of specific infection, such as cough due to pneumonia, dysuria due to urinary tract infection [24].The diagnosis of catheter related bloodstream infection (CRBSI) is often suspected clinically in a hemodialysis patient using a CVC who present with fever or chills, unexplained hypotension and no other localizing sign [32]. Mild symptoms include malaise and nausea, in the setting of a normal catheter exit site or tunnel, on physical examination. More severe symptoms include high fever with rigors, hypotension, vomiting and change in mental status, in the setting of a norma catheter exit site or tunnel, on physical 
examination. Both Gram-positive and Gram-negative organisms are responsible for BSI in patients on maintenance hemodialysis. Observed that central venous catheter-related bloodstream infection (CRBSI) was a major cause of morbidity and mortality in patients with end-stage renal disease treated with chronic hemodialysis [44]. The policy of increasing the AVF (arteriovenous fistula) prevalence beyond 50\% while minimizing the use of CVCs, dependent largely upon the timely referrals and prudently implemented pre-ESRD program - ought to have a positive impact on long-term HD outcomes [8, 9] carried out a study to evaluate the clinical outcome and costs of nosocomial and community-acquired methicillin-susceptible Staphylococcus aureus (MSSA) or methicillin-resistant S. aureus (MRSA) bloodstream infection (BSI) in patients undergoing hemodialysis. This study also highlighted differences according to the source of BSI, including costs arising from hospitalization and treatment.

\section{OBJECTIVES}

\section{A. General objective}

1. To estimate the rates of infections and clinical and microbiological evaluation of blood stream infections in patients undergoing maintenance hemodialysis.

\section{B. Specific objectives}

1. To determine risk factors for developing bloodstream infections in subjects undergoing hemodialysis.

2. To detect the causative organisms of bloodstream infections in subjects undergoing hemodialysis and determine their antibiotic sensitivity pattern.

3. To assess the rate of infection in different types of vascular accesses (Fistula and permanent catheter)

4. To observe relation between risk factors with bloodstream infection in patients receiving hemodialysis.

\section{MATERIALS AND METHODS}

This cross sectional study was conducted from January 2014 to December 2015 for a period of 2(two) years in the Department of Nephrology at Bangabandhu Sheikh Mujib Medical University, Dhaka. All adult patients underwent hemodialysis of both sexes fulfilling the inclusion and exclusion criteria were included in the study. Sample size: Due to time constrain finally 60 samples were taken.Then each patient was evaluated during each hemodialysis session for the (1) presence of bloodstream infection (BSI) using CDC (Centre for disease control) case definitions by blood cultures (Two samples were sent for cultures one from peripheral veins and another from vascular access either from fistula or from central venous catheter) and (2) clinical features.

\section{ENROLLMENT CRITERIA Inclusion criteria}

- Subjects on maintenance hemodialysis more than 3 months

- A patient with vascular access who developed the following clinical or lab criteria:

- Temperature: $>100^{\circ} \mathrm{F}$

- Heart rate:>90/minute

- Respiratory rate:>20/minute

- Peripheral white blood cell count:>12000/cmm

- Age: More than 18 years

- Sex: patients of both sexes

- Patients willing to participate in the study

\section{Exclusion criteria}

- Subjects with prophylactic antibiotic therapy

- Subjects with known infection within one month

- Acute renal failure

- Temporary venous catheter

\section{DATA ANALYSIS}

Data were recorded systematically in predesigned data collection form. Quantitative data were expressed as mean and standard deviation and qualitative data were expressed as frequency distribution and percentage. Statistical analyses were performed by using window based computer software device with Statistical Packages for Social Sciences (SPSS-21) (SPSS Inc, Chicago, IL, USA). Association between categorical variables were analyzed by chisquared test and continuous variable by unpaired student $t$-test used. For all statistical tests, $p$ value $<0.05$ as was considered as statistically significant.

\section{RESULTS AND OBSERVATION}

The present cross sectional study was conducted to estimate the rates of infections and clinical and microbiological evaluation of bloodstream infections in patients undergoing maintenance hemodialysis in the Department of Nephrology of Bangabandhu Sheikh Mujib Medical University, Dhaka. A total number of 60 patients were included in the present study. The results of the present study are as follows:

Table-I: Distribution of patients according to age group $(\mathrm{N}=60)$

\begin{tabular}{|l|c|c|}
\hline Age group & Frequency & Percentage \\
\hline$\leq 20$ & 6 & 10.0 \\
\hline $21-30$ & 17 & 28.3 \\
\hline $31-40$ & 12 & 20.0 \\
\hline $41-50$ & 7 & 11.7 \\
\hline $51-60$ & 15 & 25.0 \\
\hline$>60$ & 3 & 5.0 \\
\hline Total & 60 & 100.0 \\
\hline
\end{tabular}


[Table I] shows the text merits the description

which refer to the percent distribution of

patients/subjects in different age group.

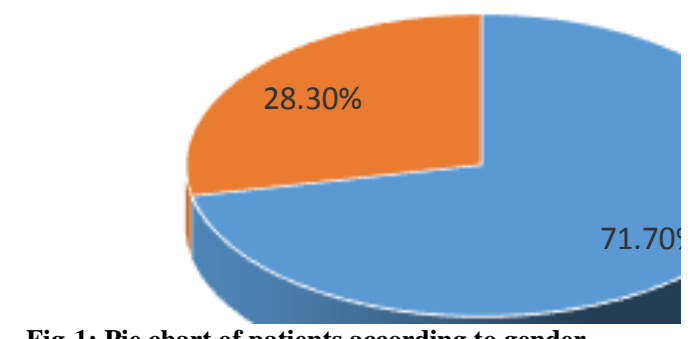

Fig-1: Pie chart of patients according to gender

Table-2: Distribution of patients according to body mass index (BMI) $(\mathrm{N}=60)$

\begin{tabular}{|l|c|c|}
\hline Nutritional status & Frequency & Percentage \\
\hline Under weight & 31 & 51.7 \\
\hline Normal & 28 & 46.7 \\
\hline Over weight & 1 & 1.7 \\
\hline Total & 60 & 100.0 \\
\hline
\end{tabular}

[Table 2] shows distribution of patients by body mass index. Most of the patients were either under weight $(51.7 \%)$ or normal $(46.7 \%)$. Body mass index $(\mathrm{kg} / \mathrm{m} 2):<18.5=$ under weight, 18.5-24.9= normal, 25 $29.9=$ over weight and $>30=$ obese

Table-3: Baseline clinical characteristics of the study sample $(n=60)$

\begin{tabular}{|c|c|c|c|}
\hline \multirow[t]{2}{*}{ Characteristic } & \multicolumn{2}{|c|}{ Bacteremia } & \multirow[t]{2}{*}{ p value } \\
\hline & $\begin{array}{c}\text { Negative } \\
(n=46)\end{array}$ & $\begin{array}{c}\text { Positive } \\
(n=14)\end{array}$ & \\
\hline Radial pulse rate /min & $99.04 \pm 8.54$ & $98.07 \pm 7.50$ & $0.703^{\#}$ \\
\hline Systolic blood pressure $(\mathrm{mm}$ of $\mathrm{Hg}$ ) & $154 \pm 22$ & $143 \pm 8$ & $0.134^{\#}$ \\
\hline Diastolic blood pressure $(\mathrm{mm}$ of $\mathrm{Hg}$ ) & $92 \pm 9$ & $86 \pm 7$ & $0.064^{\#}$ \\
\hline Temperature $\left({ }^{\circ} \mathrm{F}\right)$ & $101 \pm 1$ & $102 \pm 1$ & $0.055^{\#}$ \\
\hline Respiratory rate (b/min) & $22.6 \pm 1.6$ & $22.7 \pm 0.9$ & $0.065^{\#}$ \\
\hline Anemia & & & $0.338^{\# \#}$ \\
\hline Absent & $9(19.6)$ & $1(7.1)$ & \\
\hline Mild & $17(37.0)$ & $4(28.6)$ & \\
\hline Moderate & $20(43.5)$ & $9(64.3)$ & \\
\hline Pneumonia & $0(0.0)$ & $0(0.0)$ & \\
\hline Skin infection & $0(0.0)$ & $0(0.0)$ & \\
\hline Urinary tract infection & $0(0.0)$ & $0(0.0)$ & \\
\hline Infective endocarditis & $0(0.0)$ & $0(0.0)$ & \\
\hline Septic arthritis & $0(0.0)$ & $0(0.0)$ & \\
\hline Intra-abdominal abscess(by USG) & $0(0.0)$ & $0(0.0)$ & \\
\hline
\end{tabular}

Numeric data was expressed as Mean \pm SD and categorical data as number (percent)

[Table 3] shows that baseline clinical characteristics of both bacteremic and nonbacteremic patients are not significantly different statistically. 
Table-4: Clinico-biochemical; hematological; comorbid condition and other associated factors in subjects with or without BSI in patients receiving $\mathrm{HD}(\mathrm{N}=60)$

\begin{tabular}{|c|c|c|c|}
\hline \multirow[t]{2}{*}{ Characteristic } & \multicolumn{2}{|c|}{ Bacteremia } & \multirow[t]{2}{*}{ p value } \\
\hline & $\begin{array}{c}\text { Negative } \\
(n=46)\end{array}$ & $\begin{array}{c}\text { Positive } \\
(n=14)\end{array}$ & \\
\hline Age, yr Mean(SD) & $38 \pm 16$ & $37 \pm 12$ & $0.756^{\#}$ \\
\hline Gender & & & $0.513^{\# \#}$ \\
\hline Male & $32(69.6)$ & $11(78.6)$ & \\
\hline Female & $14(30.4)$ & $3(21.4)$ & \\
\hline Diabetes mellitus & & & $0.361^{\# \#}$ \\
\hline Yes & $12(26.1)$ & $2(14.3)$ & \\
\hline No & $34(73.9)$ & $12(85.7)$ & \\
\hline Cerebrovascular disease & & & $0.427^{\# \#}$ \\
\hline Yes & $2(4.3)$ & $0(0.0)$ & \\
\hline No & $44(95.7)$ & $14(100.0)$ & \\
\hline Vascular Access & & & $<0.001^{\# \#}$ \\
\hline AV fistula & $41(89.1)$ & $5(35.7)$ & \\
\hline Permanent catheter & $5(10.9)$ & $9(64.3)$ & \\
\hline Surgical procedure within 1 month & $3(6.5)$ & $5(35.7)$ & $0.005^{\# \#}$ \\
\hline Current iron therapy & $36(78.3)$ & $11(78.6)$ & 0.980 \\
\hline History of blood transfusion within 1 month & $20(43.5)$ & $8(57.1)$ & $0.370^{\# \#}$ \\
\hline Presence of intravenous canula & $2(4.3)$ & $0(0.0)$ & $0.427^{\# \#}$ \\
\hline S. total protein $(\mathrm{g} / \mathrm{L})$ & $63.6 \pm 6.1$ & $59.5 \pm 3.0$ & $0.020^{\#}$ \\
\hline S. Albumin (g/L) & $30.2 \pm 4.8$ & $24.6 \pm 2.4$ & $<0.001^{\#}$ \\
\hline White cell count/ cmm & $14954 \pm 4407$ & $12642 \pm 988$ & $0.058^{\#}$ \\
\hline Hemoglobin $(\mathrm{g} / \mathrm{dl})$ & $9.6 \pm 1.7$ & $9.4 \pm 1.2$ & $0.720^{\#}$ \\
\hline
\end{tabular}

[Table 4] shows that in the analysis of variables, Type of vascular access (Permanent catheter), surgical procedure within one month, low serum total protein and low serum albumin were significantly more frequently associated with bacteremia.

Table-5: Distribution of patients according to co-morbidities $(\mathrm{N}=60)$

\begin{tabular}{|l|l|l|}
\hline Co-morbidity & Frequency & Percentage \\
\hline DM & 14 & 23.3 \\
\hline Cerebrovascular disease & 2 & 3.3 \\
\hline
\end{tabular}

[Table 5] shows co-morbidities of the patients. DM was in $14(23.3 \%)$ patients and cerebrovascular disease was in $2(3.3 \%)$ patients. [DM=Diabetes mellitus]

Table-6: Distribution of patients according to anemia in two access types $(\mathrm{N}=60)$

\begin{tabular}{|c|c|c|c|c|}
\hline \multirow[t]{2}{*}{ Anemia } & \multicolumn{2}{|l|}{ Access type } & \multirow{2}{*}{$\begin{array}{l}\text { Total } \\
\text { n }(\%)\end{array}$} & \multirow{2}{*}{ p value } \\
\hline & $\begin{array}{l}\text { AV Fistula } \\
\text { n }(\%)\end{array}$ & $\begin{array}{l}\text { Central venous } \\
\text { catheter n }(\%)\end{array}$ & & \\
\hline Absent & $9(19.5)$ & $1(7.1)$ & $10(16.7)$ & \multirow[t]{4}{*}{0.525} \\
\hline Mild & $16(34.8)$ & $5(35.7)$ & $21(35.0)$ & \\
\hline Moderate & $21(45.7)$ & $8(57.2)$ & $29(48.3)$ & \\
\hline Total & $46(100.0)$ & $14(100.0)$ & $60(100.0)$ & \\
\hline
\end{tabular}

Chi-square test was done to calculate statistical significance [Table 6] shows distribution of patients according to anemia in two different access types. Anemia was moderate in $57.2 \%$ cases in central venous catheter and $45.7 \%$ cases in AV fistula. Mild anemia was presence in $35.7 \%$ cases in central venous catheter and $34.8 \%$ cases in AV fistula. Most of the patients of both groups are anemic, but there is no significant difference between two groups regarding anemia. 
Siddiqur Rahman et al; Saudi J Med Pharm Sci, Jan., 2020; 6(1): 7-19

Table-7: Hematological and biochemical variables in two groups of access type for hemodialysis $(\mathrm{N}=60)$

\begin{tabular}{|l|l|l|l|l|}
\hline & Access type & Total & \multirow{2}{*}{ p value } \\
\cline { 2 - 5 } & AV Fistula & Central venous catheter & & \\
\hline WBC $($ count/cmm) & $14652 \pm 4209$ & $13635 \pm 3229$ & $14415 \pm 4000$ & 0.410 \\
\hline Hemoglobin $(\mathrm{g} / \mathrm{dl})$ & $9.67 \pm 1.75$ & $9.41 \pm 1.13$ & $9.61 \pm 1.62$ & 0.599 \\
\hline S. Uric acid $(\mu \mathrm{mol} / \mathrm{l})$ & $410.45 \pm 108.65$ & $440.35 \pm 69.56$ & $417.67 \pm 100.87$ & 0.338 \\
\hline Plasma glucose & & & & \\
\hline Fasting $(\mathrm{mmol} / \mathrm{l})$ & $5.31 \pm 1.29$ & $5.55 \pm 0.73$ & $5.37 \pm 1.18$ & 0.508 \\
\hline 2 hours ABF & $7.29 \pm 2.27$ & $7.20 \pm 1.94$ & $7.27 \pm 2.18$ & 0.885 \\
\hline HbA1c $(\%)$ & $6.41 \pm 0.65$ & $6.46 \pm 0.78$ & $6.42 \pm 0.68$ & 0.800 \\
\hline S. total protein $(\mathrm{g} / \mathrm{l})$ & $63.79 \pm 5.87$ & $59.14 \pm 4.09$ & $62.71 \pm 5.82$ & 0.008 \\
\hline S. albumin $(\mathrm{g} / \mathrm{l})$ & $30.01 \pm 5.01$ & $25.52 \pm 3.07$ & $28.97 \pm 4.99$ & 0.003 \\
\hline
\end{tabular}

Unpaired t test was done to calculate statistical difference. [Table 7] shows laboratory findings in two different access types. Serum total protein and serum albumin were significantly higher in cases of AV fistula comparing central venous catheter type. WBC, Hemoglobin, Serum Uric acid, plasma glucose and $\mathrm{HbA} 1 \mathrm{c}$ were almost similar in both cases.

Table-8: Bloodstream infection rate of the patients $(\mathrm{N}=60)$

\begin{tabular}{|l|l|l|l|l|}
\hline & Peripheral Vein & AV fistula & Catheter & Total \\
\hline No of infection episodes & 14 & 5 & 9 & 28 \\
\hline Total patient-days & 23340 & 20390 & 2950 & 23340 \\
\hline Infection rate (per 1000 patient-days) & 0.59 & 0.24 & 3.05 & 1.19 \\
\hline
\end{tabular}

[Table 8] shows blood stream infection rate due to hemodialysis. Infection rate in fistula was
$0.24 / 1000$ patient-days, in catheter was $3.05 / 100$ patient-days and total was $1.19 / 1000$ patient-days.

Table-9: Distribution of culture positivity according to site of sampling (N=60)

\begin{tabular}{|c|c|c|c|c|c|c|c|}
\hline & \multirow[b]{2}{*}{ Total } & \multicolumn{6}{|l|}{ Positive } \\
\hline & & $\begin{array}{l}\text { Peripheral } \\
\text { Vein }\end{array}$ & Fistula & Catheter & $\begin{array}{l}\text { Catheter } \\
\text { tip }\end{array}$ & Urine & $\begin{array}{l}\text { Pleural } \\
\text { fluid }\end{array}$ \\
\hline Both peripheral vein + AV fistula & 46 & $8(13.3)$ & $5(10.8)$ & & & & \\
\hline $\begin{array}{l}\text { Both peripheral vein }+ \text { permanent } \\
\text { CV catheter }\end{array}$ & 11 & $6(54.5)$ & & $7(63.3)$ & & & \\
\hline $\begin{array}{l}\text { Both peripheral vein }+ \text { permanent } \\
\text { CV catheter }+ \text { Catheter tip culture }\end{array}$ & 3 & $0(0.0)$ & & $0(0.0)$ & $2(66.7)$ & & \\
\hline Urine culture & 7 & & & & & $0(0.0)$ & \\
\hline Pleural fluid culture & 1 & & & & & & $0(0.0)$ \\
\hline
\end{tabular}

[Table 9] shows output of culture according to culture sites. Out of $46 \mathrm{AV}$ fistula cases, culture was positive in peripheral vein $8(13.3 \%)$ cases and in fistula $5(10.8 \%)$ cases. Out of 11 permanent CV catheter cases, culture was positive in vein $6(54.5 \%)$ cases and in catheter $7(63.3 \%)$ cases. Out of 3 catheter tip cases, culture was positive in catheter tip $2(66.7 \%)$ cases. Urine and pleural fluid culture revealed no growth of bacteria.

Table-10: Names of organisms isolated from total bacterial episodes $(\mathrm{N}=60)$

\begin{tabular}{|l|l|l|}
\hline Pathogen & Frequency & Percentage \\
\hline Pseudomonas aeruginosa & 15 & 53.57 \\
\hline Acinetobacter spp & 10 & 35.71 \\
\hline Klebsiella spp. & 3 & 10.71 \\
\hline
\end{tabular}

[Table 10] shows among 28 bacterial episodes Pseudomonas aeruginosa were $53.57 \%$, Acinetobacter spp. were $35.71 \%$ and Klebsiella spp. were $10.71 \%$ 
Table-11: Outcome of bacterial culture in samples from AV fistula and central venous catheter $(\mathrm{N}=60)$

\begin{tabular}{|l|l|l|l|}
\hline \multirow{2}{*}{ Culture } & \multicolumn{2}{|l|}{ Access type } & \multirow{2}{*}{ p value } \\
\cline { 2 - 3 } & $\begin{array}{l}\text { AV Fistula } \\
(\mathrm{n}=46)\end{array}$ & Central venous catheter $(\mathrm{n}=14)$ & \\
\hline No growth & $41(89.1)$ & $5(35.7)$ & $<0.001$ \\
\hline Positive & $5(10.9)$ & $9(64.3)$ & \\
\hline & & & \\
\hline
\end{tabular}

Chi-square test was done to calculate statistical association.

[Table 11] shows comparison of growth between AV fistula and central venous catheter site. Positive growth was significantly higher in central venous catheter $9(64.3 \%)$ than that of AV fistula 5 $(10.9 \%)$ cases.

Table-12: Organisms isolated from peripheral vein (N=60)

\begin{tabular}{|l|l|l|}
\hline Gram-negative bacteria & Frequency & Percentage \\
\hline Acinetobacter spp & 4 & 6.7 \\
\hline Pseudomonas aeruginosa & 10 & 16.7 \\
\hline
\end{tabular}

[Table 12] shows organisms isolated from peripheral vein. Acinetobacter spp was present in $6.7 \%$ cases and Pseudomonas aeruginosa was present in $16.7 \%$ cases.

Table-13: Peripheral vein sensitivity pattern of Acinetobacter spp. $(\mathrm{N}=60)$

\begin{tabular}{|l|l|l|}
\hline Antibiotics & Sensitive & Resistant \\
\hline Piperacillin + Tazobactum & $4(100.0)$ & \\
\hline Cefuroxime & & $2(100.0)$ \\
\hline Ceftazidime & & $3(100.0)$ \\
\hline Ceftriaxone & & $1(100.0)$ \\
\hline Imipenem & $1(33.3)$ & $2(66.7)$ \\
\hline Amikacin & $4(100.0)$ & \\
\hline Netilmicin & $3(75.0)$ & $1(25.0)$ \\
\hline Amoxicillin & & $3(100.0)$ \\
\hline Ciprofloxacin & $4(100.0)$ & \\
\hline Co-trimoxazole & $3(75.0)$ & $1(25.0)$ \\
\hline Colistin & $4(100.0)$ & \\
\hline Cefotaxime & & $2(100.0)$ \\
\hline Ticarcillin & & $2(100.0)$ \\
\hline Aztreonam & & $4(100.0)$ \\
\hline
\end{tabular}

[Table 13] shows sensitivity pattern of Acinetobacter spp. Piperacillin + Tazobactum(4/4), Amikacin(4/4), Ciprofloxacin(4/4) and Colistin(4/4) antibiotics were sensitive in $100 \%$ cases. Imipenem
(1/3) was sensitive in $33.3 \%$ cases. Netilmicin(3/4) and co-trimoxazole(3/4) was sensitive in $75 \%$ cases. Cefuroxime (2/2), Ceftazidime (3/3), Ceftriaxone (1/1), Amoxicillin (3/3), cefotaxime(2/2), Ticarcillin(2/2) and Aztreonam(4/4) were resistant $100 \%$ cases.

Table-14: Peripheral vein sensitivity pattern of Pseudomonas aeruginosa $(\mathrm{N}=60)$

\begin{tabular}{|l|l|l|}
\hline Antibiotics & Sensitive & Resistant \\
\hline Piperacillin + Tazobactum & $10(100)$ & \\
\hline Cefuroxime & & $5(100)$ \\
\hline Ceftazidime & & $9(100)$ \\
\hline Ceftriaxone & & $3(100)$ \\
\hline Cefepime & & $5(100)$ \\
\hline Imipenem & $2(50)$ & $2(50)$ \\
\hline Amikacin & $9(90)$ & $1(10)$ \\
\hline Netilmicin & $6(60)$ & $4(40)$ \\
\hline Gentamicin & $5(50)$ & $5(50)$ \\
\hline Amoxicillin & & $3(100)$ \\
\hline Ciprofloxacin & $10(100)$ & \\
\hline Co-trimoxazole & $2(66.7)$ & $1(33.3)$ \\
\hline Colistin & $10(100)$ & \\
\hline Cefotaxime & $2(100)$ & \\
\hline Ticarcillin & $6(60)$ & $4(40)$ \\
\hline Aztreonam & $8(100)$ & \\
\hline
\end{tabular}

[Table 14] shows sensitivity pattern of Pseudomonas aeruginosa. Piperacillin + Tazobactum (10/10), Ciprofloxacin (10/10), Colistin(10/10), Cefotaxime (2/2) and Aztreonam(8/8) antibiotics were sensitive in $100 \%$ cases. Imipenem (2/4) was sensitive in $50 \%$ cases, Amikacin (9/10) was sensitive in $90 \%$ cases, Netilmicin (6/10) was sensitive in $60 \%$ cases, Gentamicin (5/10) was sensitive in $50 \%$ cases, cotrimoxazole $(2 / 3)$ was sensitive in $66.7 \%$ cases, Ticarcillin(6/10) was sensitive in $60 \%$ cases. Cefuroxime (5/5), Ceftazidime (9/9), Ceftriaxone (3/3), Cefepime (5/5) and Amoxicillin(3/3) were resistant in $100 \%$ cases.

Table-15: Organisms isolated from vascular access sites $(\mathrm{N}=60)$

\begin{tabular}{|l|l|l|l|}
\hline \multirow{2}{*}{ Gram-negative bacteria } & Access type & \multirow{2}{*}{ p value } \\
\cline { 2 - 4 } & $\begin{array}{l}\text { AV Fistula n } \\
(\%)\end{array}$ & $\begin{array}{l}\text { Central } \\
\text { catheter n (\%) }\end{array}$ & \\
\hline Klebsiella spp. & $0(0.0)$ & $3(21.4)$ & 0.001 \\
\hline Acinetobacter spp & $3(6.5)$ & $3(21.4)$ & 0.104 \\
\hline Pseudomonas aeruginosa & $2(4.3)$ & $3(21.4)$ & 0.043 \\
\hline
\end{tabular}

Chi-square test was done to measure the level of significance [Table 15] shows organisms isolated from vascular access site. Acinetobacter spp were present in 3(6.5\%) cases and Pseudomonas aeruginosa 
Siddiqur Rahman et al; Saudi J Med Pharm Sci, Jan., 2020; 6(1): 7-19

were present in $2(4.3 \%)$ cases in AV fistula. Klebsiella spp., Acinetobacter spp and Pseudomonas aeruginosa all were present in 3 (21.43) cases in Central venous catheter.

Table-16: Central venous catheter sensitivity pattern of Klebsiella spp. $(\mathrm{N}=60)$

\begin{tabular}{|c|c|c|c|c|}
\hline $\begin{array}{c}\text { Antibiotics } \\
\text { (sensitive) }\end{array}$ & AV Fist & ula & \multicolumn{3}{|c|}{$\begin{array}{c}\text { Central venous catheter } \\
\text { Sensitive Resistant }\end{array}$} \\
\cline { 4 - 5 } & & & & $2(100.0)$ \\
\hline Cefuroxime & & & & $2(100.0)$ \\
\hline Ceftazidime & & & & $2(100.0)$ \\
\hline Ceftriaxone & & & $2(100.0)$ & \\
\hline Imipenem & & & $1(50.0)$ & $1(50.0)$ \\
\hline Amikacin & & & $2(100.0)$ & \\
\hline Gentamicin & & & & $2(100.0)$ \\
\hline Amoxicillin & & & $2(100.0)$ & \\
\hline Ciprofloxacin & & & & $2(100.0)$ \\
\hline Co-trimoxazole & & & $2(100.0)$ & \\
\hline Colistin & & & $1(50.0)$ & $1(50.0)$ \\
\hline Nalidixic & & & $1(50.0)$ & $1(50.0)$ \\
\hline Cefradine & & & & \\
\hline
\end{tabular}

[Table 16] shows sensitivity pattern of Klebsiella spp.: Imipenem (2/2), Gentamicin (2/2), Ciprofloxacin(2/2) and Colistin(2/2) antibiotics were sensitive in $100 \%$ cases. Amikacin (1/1), Cefradine(1/1) and Nalidixic acid(1/1) were sensitive in $50.0 \%$ cases. Cefuroxime (2/2), Ceftazidime (2/2), Ceftriaxone (2/2), Amoxicillin (2/2) and Co-trimoxazole(2/2) antibiotics were resistant in $100 \%$ cases.

Table-17: Sensitivity pattern of Acinetobacter spp. (N=60)

\begin{tabular}{|l|l|l|l|l|}
\hline \multirow{2}{*}{ Antibiotics (sensitive) } & Access type & & \multicolumn{2}{l|}{ Central venous catheter Sensitive } \\
\cline { 2 - 5 } & $\begin{array}{l}\text { AV Fistula } \\
\text { Sensitive Resistant }\end{array}$ & & \multicolumn{2}{l|}{ Restant } \\
\hline Piperacillin + Tazobactum & $3(100.0)$ & & $3(100.0)$ & $3(100.0)$ \\
\hline Cefuroxime & & $3(100.0)$ & & $1(100.0)$ \\
\hline Cefixime & & $3(100.0)$ & & $3(100.0)$ \\
\hline Ceftazidime & & $3(100.0)$ & & $2(100.0)$ \\
\hline Ceftriaxone & & $3(100.0)$ & & $1(33.3)$ \\
\hline Imipenem & $2(66.7)$ & $2(66.7)$ & \\
\hline Amikacin & $1(33.3)$ & $3(100.0)$ & \\
\hline Netilmicin & $3(100.0)$ & & $2(100.0)$ & $3(100.0)$ \\
\hline Gentamicin & $3(100.0)$ & $2(66.7)$ & & \\
\hline Amoxicillin & & & $3(100.0)$ & \\
\hline Ciprofloxacin & $1(33.3)$ & $1(33.3)$ & $1(100.0)$ & $2(100.0)$ \\
\hline Co-trimoxazole & $3(100.0)$ & & $2(100.0)$ & \\
\hline Colistin & $2(66.7)$ & $2(66.7)$ & & \\
\hline Aztreonam & $3(100.0)$ & $2(66.7)$ & & \\
\hline Nalidixic & $1(33.3)$ & $3(100.0)$ & & \\
\hline Cefotaxime & $1(33.3)$ & & & \\
\hline
\end{tabular}

[Table 17] shows sensitivity pattern of Acinetobacter spp. in AV fistula and central venus catheter. In AV fistula: Colistin(3/3), Amikacin(3/3), Netilmicin(3/3), Ciprofloxacin(3/3) and Piperacillin + Tazobactum(3/3) were sensitive in $100 \%$ cases. Cefuroxime (3/3), Cefixime(3/3), Ceftazidime(3/3), Ceftriaxone(3/3) and Cefotaxime(3/3) were resistant in $100 \%$ cases. Imipenem (2/3), Amoxicillin(2/3), Aztreonam(2/3) and Nalidixic(2/3) were resistant in
$66.7 \%$ cases. In central venous catheter: Colistin(2/2), Amikacin(3/3), Netilmicin(2/2), Ciprofloxacin(3/3), Gentamicin(1/1), Co-trimoxazole(1/1) and Piperacillin + Tazobactum(3/3) were sensitive in $100 \%$ cases. Imipenem (2/3) was sensitive in $66.7 \%$ cases. Cefuroxime (3/3), Cefixime(1/1), Ceftazidime(3/3), Ceftriaxone(2/2), Amoxicillin(3/3) and Aztreonam(2/2) were resistant in $100 \%$ cases. 
Table 18: Sensitivity pattern of Pseudomonas aeruginosa $(\mathrm{N}=60)$

\begin{tabular}{|l|l|l|l|l|}
\hline \multirow{2}{*}{ Antibiotics (sensitive) } & Access type & & \multicolumn{3}{|l|}{} \\
\cline { 2 - 5 } & AV Fistula & & \multicolumn{2}{|l|}{ Central venous catheter } \\
\cline { 2 - 5 } & Sensitive & Resistant & Sensitive & Resistant \\
\hline Piperacillin + Tazobactum & $2(100.0)$ & & $2(100.0)$ & \\
\hline Cefixime & & & & $2(100.0)$ \\
\hline Ceftazidime & & $2(100.0)$ & & $2(100.0)$ \\
\hline Ceftriaxone & & & & $2(100.0)$ \\
\hline Cefepime & & $2(100.0)$ & & \\
\hline Imipenem & & $2(100.0)$ & $1(50.0)$ & $1(50.0)$ \\
\hline Amikacin & $2(100.0)$ & & $2(100.0)$ & \\
\hline Netilmicin & $1(50.0)$ & $1(50.0)$ & $1(50.0)$ & $1(50.0)$ \\
\hline Gentamicin & $1(50.0)$ & $1(50.0)$ & $1(50.0)$ & $1(50.0)$ \\
\hline Amoxicillin & & & & $2(100.0)$ \\
\hline Ciprofloxacin & $2(100.0)$ & & $2(100.0)$ & \\
\hline Colistin & $2(100.0)$ & & $2(100.0)$ & \\
\hline Ticarcillin & $1(50.0)$ & $1(50.0)$ & $1(50.0)$ & $1(50.0)$ \\
\hline Aztreonam & & $2(100.0)$ & $1(50.0)$ & $1(50.0)$ \\
\hline
\end{tabular}

[Table 18] shows sensitivity pattern of organisms (Pseudomonas aeruginosa) isolated from vascular access site: In AV fistula: Piperacillin + Tazobactum(2/2), Amikacin(2/2), Ciprofloxacin(2/2) and Colistin(2/2) were sensitive in $100 \%$ cases. Ticarcillin(1/1), Gentamicin(1/1) and Netilmicin(1/1) were sensitive in $50 \%$ cases. Ceftazidime (2/2), Cefepime(2/2), Aztreonam(2/2) and Imipenem(2/2) were resistant in $100 \%$ cases. In Central venous catheter: Piperacillin + Tazobactum(2/2), Amikacin(2/2), Ciprofloxacin(2/2) and Colistin(2/2) were sensitive in $100 \%$ cases. Ceftazidime (2/2), Cefixime(2/2), Ceftriaxone(2/2) and Amoxicillin(3/3) were resistant in $100 \%$ cases. Imipenem $(1 / 2$, )Ticarcillin(1/2), Gentamicin(1/2), Netilmicin(1/2) and Aztreonam(1/2) were resistant in $66.7 \%$ cases.

\section{Table-19: Organisms isolated from catheter tip}

$$
(\mathrm{N}=60)
$$

\begin{tabular}{|l|l|l|}
\hline Gram-negative bacteria & Frequency & Percentage \\
\hline Klebsiella spp. & 1 & 33.3 \\
\hline Pseudomonas aeruginosa & 1 & 33.3 \\
\hline
\end{tabular}

[Table 19] shows organisms isolated from catheter tip. Klebsiella spp. was present in $33.3 \%$ case and Pseudomonas aeruginosa was present in $33.3 \%$ case.

Table-20: Catheter tip sensitivity pattern of Klebsiella spp. $(\mathrm{N}=60)$

\begin{tabular}{|l|l|l|}
\hline Antibiotics & Sensitive & Resistant \\
\hline Cefuroxime & & $1(100.0)$ \\
\hline Ceftazidime & & $1(100.0)$ \\
\hline Ceftriaxone & & $1(100.0)$ \\
\hline Imipenem & & $1(100.0)$ \\
\hline Amikacin & & $1(100.0)$ \\
\hline Netilmicin & $1(100.0)$ \\
\hline Gentamicin & $1(100.0)$ & \\
\hline Amoxicillin & & $1(100.0)$ \\
\hline Ciprofloxacin & $1(100.0)$ & \\
\hline Colistin & $1(100.0)$ & \\
\hline Nalidixic & $1(100.0)$ & \\
\hline
\end{tabular}

[Table 20] shows sensitivity pattern of Klebsiella spp. Gentamicin, Ciprofloxacin, Colistin and Nalidixic antibiotics were sensitive in Klebsiella spp bacteria but Cefuroxime, Ceftazidime, Ceftriaxone, Imipenem, Amikacin, Netilmicin and Amoxicillin antibiotics were resistant in Klebsiella spp bacteria.

Table-21: Catheter tip sensitivity pattern of Pseudomonas aeruginosa $(\mathrm{N}=60)$

\begin{tabular}{|l|l|l|}
\hline Antibiotics & Sensitive & Resistant \\
\hline Piperacillin + Tazobactum & $1(100.0)$ & \\
\hline Cefepime & $1(100.0)$ & \\
\hline Imipenem & $1(100.0)$ & \\
\hline Amikacin & $1(100.0)$ & \\
\hline Netilmicin & $1(100.0)$ & \\
\hline Gentamicin & $1(100.0)$ & \\
\hline Ciprofloxacin & $1(100.0)$ & \\
\hline Colistin & $1(100.0)$ & \\
\hline Ticarcillin & $1(100.0)$ & \\
\hline
\end{tabular}

[Table 21] shows sensitivity pattern of Pseudomonas aeruginosa. Tazobactum, Cefepime, Imipenem, Amikacin, Netilmicin, Gentamicin, Ciprofloxacin, Colistin and Ticarcillin antibiotics were sensitive in Pseudomonas aeruginosa.

\section{DISCUSSION}

Bloodstream infection (BSI) is the leading cause of hospitalization and the second most common cause of death (after cardiovascular death) among patients receiving regular hemodialysis [29]. In the present cross sectional study the rates of infections was estimated and clinical and microbiological evaluation of bloodstream infections in patients undergoing hemodialysis was done. In this study mean (SD) age of the study population was $38.26(15.26)$ within the range of 16-70 years. Maximum (28.3\%) patients were in age group 21-30 years followed by (25.0\%) were in age group 51-60 years. Mean age was comparatively higher in others studies [30, 31]. Patient at any age may require hemodialysis. Males $(71.7 \%)$ were predominant 
than females (28.3\%). Male female ratio was 2.52:1. Any gender may require hemodialysis [30, 31]. Females are associated with more bacteremia than the males [23, 30]. Most of the patients were either under weight $(51.7 \%)$ or normal $(46.7 \%)$. Evaluation of baseline clinical characteristics such as pulse, blood pressure, respiratory rate, temperature and anemia, body mass index of both bacteremic and nonbacteremic patients revealed no significant difference. During further evaluation no patient was found to have urinary tract infection, pneumonia, infective endocarditis, skin infection, septic arthritis [32]. Stated that hemodialysis patient may be suspected for bloodstream infection who present with fever or chills, unexplained hypotension, and no other localizing sign [24]. Found pneumonia $0.84 / 100$ patient-month, urinary tract infection 0.29 / 100 patient-month, wound infection $1.29 / 100$ patientmonth for all access. In this study risk factors for bloodstream infections were searched and found that tunneled central venous catheter, surgical procedure within one month, low serum total protein and low serum albumin were significantly associated with bacteremia [33]. Showed the risk of bacteremia was highest in hemodialysis patients using central venous catheter as vascular access and the incidence rate of CRBSI was 2.5 to 5.5 cases/1000 catheter days. Risk factors described in other studies include: older age [42] higher total intravenous iron dose $[38,39]$ increased recombinant human erythropoietin dose [6], lower hemoglobin level $[39,40]$ low serum albumin level $[42$, 24]; Diabetes mellitus [32] and recent surgery [24]. Regarding co-morbidities of the patients, DM was in 14 $(23.3 \%)$ patients and cerebrovascular disease was in 2 (3.3\%) patients [31]. Found DM (32.0\%) in their study [30]. Found DM (39.0\%) and Coronary disease $(18.8 \%)$. Most of the patients were found to have mild to moderate anemia. Anemia was moderate in $57.2 \%$ cases in central venous catheter and $45.7 \%$ cases in AV fistula. Mild anemia was presence in $35.7 \%$ cases in central venous catheter and $34.8 \%$ cases in AV fistula [40]. Showed anemia were significantly associated with bacteremia. Bloodstream infections rate due to hemodialysis were recorded. In this study, total 28 bacteria were identified at a infection rate of 1.19/1000 patient-days. Of the 28 bacteria 5 involved permanent fistula (0.24/1000 patient-days); and 9 involved permanent-tunneled central venous catheter $(3.05 / 1000$ patient-days). There were 14 bloodstream infections (0.59/1000 patient days). In the study of [30] the BSI rate was 0.52 per 1000 patient-days. Of the 148 episodes, 34 occurred in patients with permanent fistula (0.18/1000 patient-days); 19 in patients with grafts (0.39/1000 patient-days); 28 in patients with permanent tunneled central catheters (1.03/1000 patient-days); and 67 in those with temporary catheter (3.18/1000 patientdays) [43]. Identified a total of 109 infections, for a rate of 11.32/1000 dialysis sessions (ds). Of the 109, 23 involved permanent fistulae or grafts $(4.23 / 1000 \mathrm{ds}) ; 18$ involved permanent-tunneled central catheter infections
$(10.1 / 1000 \mathrm{ds})$; and 68 involved temporary-catheter infections (28.23/1000 ds). There were 38 bloodstream infections (3.95/1000 ds). Positive growth was significantly higher in central venous catheter 9 (64.3\%) than that of AV fistula $5(10.9 \%)$ cases. In their study [40, 41] described hemodialysis catheter were major risk factors for bacteremia particularly when compared to synthetic graft or native arteriovenous fistula. Out of $46 \mathrm{AV}$ fistula cases, culture was positive in peripheral veins $8(13.3 \%)$ cases and in fistula 5 (10.8\%) cases. Out of 11 permanent CV catheter cases, culture was positive in peripheral veins $6(54.5 \%)$ cases and in catheter $7(63.3 \%)$ cases. Out of 3 catheter tip cases, culture was positive in catheter tip $2(66.7 \%)$ cases. Out of 7 Urine for $\mathrm{C} / \mathrm{S} \& 1$ pleural fluid culture revealed no growth. Regarding source of bacteremia: 3 cases were primary bacteremia and rests of the cases were either due to permanent catheter or due to AV fistula [30]. Found primary bacteremia 51\%, CRBSI $28 \%$ and $21 \%$ secondary to other sources. Regarding causative organisms causing bacteremia: All identified bacteremia were gram-negative and the high prevalence of gram-negative bacteria may be due to immunocompromised states of the patients, contaminated infusate and misuse of antibiotics. Pseudomonas aeruginosa were $53.57 \%$, Acinetobacter spp. were $35.71 \%$ and Klebsiella spp. were $10.71 \%$. In their study, $\mathrm{Al}$ muneef et al. (2006) noted a total 50 CRBSI episodes; among them $48 \%$ were polymicrobial, $32 \%$ were due to gram-negative bacilli and $10 \%$ due to gram-positive organisms. The most common organisms isolated were Klebsiella $16 \%$, coaglase- negative staphylococci $14 \%$ and Pseudomonas aeruginosa $11 \%$ [30]. Noted gram-negative bacteria as a cause of bloodstream infections in 33\% cases. Among them Escherichia coli were 39\%, Klebsiella spp. were $17 \%$, Acinetobacter spp. were $14 \%$ and Enterobacter spp. were $14 \%$. Regarding organisms isolated from peripheral veins, Acinetobacter spp. was present in 6.7\% cases and Pseudomonas aeruginosa was present in $16.7 \%$ cases. Sensitivity pattern of Acinetobacter spp and Pseudomonas spp. in peripheral vein were recored. Most sensitive antibiotics were Piperacillin + Tazobactum, Amikacin, Ciprofloxacin and Colistin. Moderately sensitive antibiotics were Imipenem, Cotrimoxazole and Netilmicin. Most resistant antibiotics were Cefuroxime, Ceftazidime, Ceftriaxone, Amoxicillin, cefotaxime, Gentamicin and Ticarcillin. Regarding organisms isolated from vascular access site, Acinetobacter spp in 3(6.5\%) and Pseudomonas aeruginosa were present in 2(4.3\%) cases in AV fistula. In Central venous catheter. Klebsiella spp in 3(21.4\%). Acinetobacter spp in 3(21.4\%) and Pseudomonas aeruginosa in 3(21.4\%) cases were present. Sensitivity pattern of Klebsiella spp. in central venous catheter: Imipenem, Gentamicin, Ciprofloxacin and Colistin antibiotics were sensitive in $100 \%$ cases. Cefuroxime, Ceftazidime, Ceftriaxone, Amoxicillin and Cotrimoxazole antibiotics were resistant in $100 \%$ cases. 
Sensitivity pattern of Acinetobacter spp. in AV fistula and central venus catheter. Most sensitive antibiotics were: Colistin, Amikacin, Netilmicin, Ciprofloxacin and Piperacillin + Tazobactum. Moderately sensitive antibiotics were Imipenem, Aztreonam and most resistant antibiotics were Cefuroxime, Cefixime, Ceftazidime, Ceftriaxone and Cefotaxime. Sensitivity pattern of organisms (Pseudomonas aeruginosa) isolated from vascular access sites: Most sensitive antibiotics were Piperacillin + Tazobactum, Amikacin, Ciprofloxacin and Colistin. Most resistant antibiotics were Ceftazidime, Cefepime, Aztreonam and Imipenem. Regarding organisms isolated from catheter tip, Klebsiella spp. was present in $33.3 \%$ case and Pseudomonas aeruginosa was present in $33.3 \%$ case. Sensitivity pattern of Klebsiella spp.in catheter tip: Gentamicin, Ciprofloxacin, Colistin and Nalidixic antibiotics were sensitive in Klebsiella spp bacteria but Cefuroxime, Ceftazidime, Ceftriaxone, Imipenem, Amikacin, Netilmicin and Amoxicillin antibiotics were resistant in Klebsiella spp bacteria. Sensitivity pattern of Pseudomonas aeruginosa in catheter tip: Tazobactum, Cefepime, Imipenem, Amikacin, Netilmicin, Gentamicin, Ciprofloxacin, Colistin and Ticarcillin antibiotics were sensitive in Pseudomonas aeruginosa. Arterial catheters used for hemodynamic monitoring $(0.8 \%, 1.7$ per 1000 catheter-days) and peripherally inserted central catheters used in hospitalized patients $(2.4 \%, 2.1$ per 1000 catheter-days) posed risks approaching those seen with short-term conventional CVCs used in the Intensive care unit. Surgically implanted long-term central venous devices-cuffed and tunneled catheters $(22.5 \%, 1.6$ per 1000 IVD-days) and central venous ports $(3.6 \%, 0.1$ per 1000 IVD-days)--appear to have high rates of infection when risk is expressed as BSIs per 100 IVDs but actually pose much lower risk when rates are expressed per 1000 IVD-days. The use of cuffed and tunneled dual lumen CVCs rather than noncuffed, nontunneled catheters for temporary hemodialysis and novel preventive technologies, such as CVCs with antiinfective surfaces, was associated with considerably lower rates of catheter-related BSI. Regardless of the repeated reservations raised by countless researchers with reference to the use of catheters as vascular access for hemodialysis (HD), central venous catheters (CVCs) remain irreplaceable tools of the modern dialysis delivery system as a reliable option for the clinical situations requiring instant access to circulation, for various reasons. Patients on long-term hemodialysis are therefore at a significantly high risk for catheter related bloodstream infections (CRBSI) and ensuing serious complications.

\section{CONCLUSIONS}

In hemodialysis patients, the risk of bacteremia can be stratified according to vascular access type. Infections rates were highest in hemodialysis patients with central venous catheter access, compared with rate in those with AV fistula. Low serum total protein, low serum albumin, low hemoglobin and poor nutrition are risk factors for bloodstream infections (BSI). To reduce the rates of bloodstream infections careful clinical evaluation, limitation of the catheter use as vascular access, improvement of nutritional status, correction of anemia and hypoalbuminaemia are essential. BSI is responsible for mortality, morbidity, cost and hazards of the patients. The $1^{\text {st }}$ step to minimize BSI is to dialysis through AV fistula, next step is to recognition of risk factors and their management and the $3^{\text {rd }}$ step is to early recognition and treatment $\mathrm{BSI}$.

\section{REFERENCES}

1. Nahas, M., \& Khwaja, A. (2015). Epidemiology, natural history, and pathophysiology of chronic kidney disease. In Johnson, R. Feehaly, J., \& Floege, J. (EDS.). Comprehensive Clinical Nephrology. $5^{\text {th }}$ ed. Philadelphia: Elsevier, pp. 919

2. Lameire, N., Van Biesen, W. (2010). 'The initiation of renal-replacement therapy--just-intime delivery', N Engl J Med; 363(7): pp. 678-680.

3. KDIGO. (2013). Kidney Int Supp, 3(1), 1-150

4. Beathard, G. (1999). 'Management of bacteremia associated with tunneled-cuffed hemodialysis catheters', J Am Soc Nephrol, 10(5): pp. 10451049

5. Quarello, F. Forneris, G. Borca, M. Pozzato, M. (2006). 'Do central venous catheters have advantages over arteriovenous fistulas or grafts?', $J$ Nephrol; 19, 265-279

6. Weijmer, M. C., Vervloet, M. G., \& ter Wee, P. M. (2004). Compared to tunnelled cuffed haemodialysis catheters, temporary untunnelled catheters are associated with more complications already within 2 weeks of use. Nephrology Dialysis Transplantation, 19(3), 670-677.

7. Thomson, P., Stirling, C. M., Geddes, C. C., Morris, S. T., \& Mactier, R. A. (2007). Vascular access in haemodialysis patients: a modifiable risk factor for bacteraemia and death. QJM: $A n$ International Journal of Medicine, 100(7), 415422.

8. Saxena, A. K., \& Panbotra, B. R. (2005). Haemodialysis catheter-related bloodstream infections: current treatment options and strategies for prevention. Swiss medical weekly, 135(9-10), 127-138.

9. Greiner, W., Rasch, A., Köhler, D., Salzberger, B., Fätkenheuer, G., \& Leidig, M. (2007). Clinical outcome and costs of nosocomial and communityacquired Staphylococcus aureus bloodstream infection in haemodialysis patients. Clinical microbiology and infection, 13(3), 264-268.

10. Pendse, S. Singh, A. Zawada, E. (2008). Initiation of Dialysis. In: Handbook of Dialysis. 4th ed. New York, NY: pp.14-21

11. Brundage, D. (1992). Renal Disorders. St. Louis, MO: Mosby 
12. Hamilton, R.W. (1998). "Principles of Dialysis: Diffusion, Convection, and Dialysis Machines" In: Schrier RW (ed) Atlas of Diseases of the Kidney, Current Medicine, Blackwell Science, Philadelphia.

13. New York Times. (2009). 'Willem Kolff, Doctor Who Invented Kidney and Heart Machines, Dies at 97'.

http://www.nytimes.com/2009/02/13/health/13kolff .html?pagewanted=all;

14. Mosby's Dictionary of Medicine, Nursing, \& Health Professions. 7th ed. St. Louis, MO; Mosby: 2006

15. Ahmad, S. Misra, M. Hoenich, N. Daugirdas, J. (2008). Hemodialysis Apparatus. In: Handbook of Dialysis; 4th ed. New York, NY; pp. 59-78

16. Pfuntner, A. Wier, L. Stocks, C. (2013). Most Frequent Procedures Performed in U.S. Hospitals, 2011. HCUP Statistical Brief \#165. October 2013. Agency for Healthcare Research and Quality, Rockville, MD

17. Cheesbrough, J. S., Finch, R. G., \& Burden, R. P. (1986). A prospective study of the mechanisms of infection associated with hemodialysis catheters. Journal of Infectious Diseases, 154(4), 579-589.

18. De Cicco, M., Campisi, C., \& Matovic, M. (2003). Central venous catheter-related bloodstream infections: pathogenesis factors, new perspectives in prevention and early diagnosis.

19. Garner, J. Jarvis, W. Emori, T. (1988). 'CDC definitions for nosocomial infections'. Am J Infect Control; 16, 128-140.

20. Adeniyi, O. Tzamaloukas, A. (2003). 'Relation between access-related infection and pre-infection serum albumin concentration in patients on chronic hemodialyses. Hemodial Int; 7: 304-310.

21. Descamps-Latscha, B. Drüeke, T. Witko-Sarat, V. (2001). 'Dialysis-induced oxidative: stress: biological aspects, clinical consequences, and therapy'. Semin Dial; 14, 193-199.

22. Meier, P., Dayer, E., Blanc, E., \& Wauters, J. P. (2002). Early $\mathrm{T}$ cell activation correlates with expression of apoptosis markers in patients with end-stage renal disease. Journal of the American Society of Nephrology, 13(1), 204-212.

23. Stevenson, K. B., Hannah, E. L., Lowder, C. A., Adcox, M. J., Davidson, R. L., Mallea, M. C., ... \& Wagnild, J. P. (2002). Epidemiology of hemodialysis vascular access infections from longitudinal infection surveillance data: predicting the impact of NKF-DOQI clinical practice guidelines for vascular access. American Journal of Kidney Diseases, 39(3), 549-555.

24. Tokars, J. I., Miller, E. R., \& Stein, G. (2002). New national surveillance system for hemodialysisassociated infections: initial results. American journal of infection control, 30(5), 288-295.
25. Calligaro, K. D., Veith, F. J., Schwartz, M. L., Dougherty, M. J., \& DeLaurentis, D. A. (1995). Differences in early versus late extracavitary arterial graft infections. Journal of vascular surgery, 22(6), 680-688.

26. Ryan, S. V., Calligaro, K. D., Scharff, J., \& Dougherty, M. J. (2004). Management of infected prosthetic dialysis arteriovenous grafts. Journal of vascular surgery, 39(1), 73-78.

27. Padberg, F. T., Smith, S. M., \& Eng, R. H. (1995). Accuracy of disincorporation for identification of vascular graft infection. Archives of Surgery, 130(2), 183-187.

28. Schwab, S. J., Raymond, J. R., Saeed, M., Newman, G. E., Dennis, P. A., \& Bollinger, R. R. (1989). Prevention of hemodialysis fistula thrombosis. Early detection of venous stenoses. Kidney international, 36(4), 707-711.

29. Lukowsky, L. R., Kheifets, L., Arah, O. A., Nissenson, A. R., \& Kalantar-Zadeh, K. (2012). Patterns and predictors of early mortality in incident hemodialysis patients: new insights. American journal of nephrology, 35(6), 548-558.

30. Fysaraki, M., Samonis, G., Valachis, A., Daphnis, E., Karageorgopoulos, D. E., Falagas, M. E., ... \& Kofteridis, D. P. (2013). Incidence, clinical, microbiological features and outcome of bloodstream infections in patients undergoing hemodialysis. International journal of medical sciences, 10(12), 1632.

31. Fram, D., Okuno, M. F. P., Taminato, M., Ponzio, V., Manfredi, S. R., Grothe, C., \& Barbosa, D. (2015). Risk factors for bloodstream infection in patients at a Brazilian hemodialysis center: a casecontrol study. BMC infectious diseases, 15(1), 158.

32. Allon, M., Depner, T. A., Radeva, M., Bailey, J., Beddhu, S., Butterly, D., \& Lewis, J. A. (2003). Impact of dialysis dose and membrane on infection-related hospitalization and death: results of the HEMO Study. Journal of the American Society of Nephrology, 14(7), 1863-1870.

33. Lee, L. Y., Miyamoto, Y. J., McIntyre, B. W., Höök, M., McCrea, K. W., McDevitt, D., \& Brown, E. L. (2002). The Staphylococcus aureus Map protein is an immunomodulator that interferes with $\mathrm{T}$ cell-mediated responses. The Journal of clinical investigation, 110(10), 1461-1471.

34. Taylor, G., Gravel, D., Johnston, L., Embil, J., Holton, D., Paton, S., \& Canadian Hospital Epidemiology Committee. (2004). Incidence of bloodstream infection in multicenter inception cohorts of hemodialysis patients. American journal of infection control, 32(3), 155-160.

35. Krishnasami, Z., Carlton, D., Bimbo, L., Taylor, M. E., Balkovetz, D. F., Barker, J., \& Allon, M. (2002). Management of hemodialysis catheterrelated bacteremia with an adjunctive antibiotic 
lock solution. Kidney international,61(3), 11361142.

36. Salman, L., Asif, A., \& Allon, M. (2015).Venous catheter infections and other complications. In Daugirdas, J. Blake, P. and Ing, T. (eds.). Handbook of dialysis. $5^{\text {th }}$ ed. Philadelphia: Wolters kluwer

37. Grothe, C., Belasco, A. G. D. S., Bittencourt, A. R. D. C., Vianna, L. A. C., Sesso, R. D. C. C., \& Barbosa, D. A. (2010). Incidence of bloodstream infection among patients on hemodialysis by central venous catheter. Revista latino-americana de enfermagem, 18(1), 73-80.

38. Jean, G., Charra, B., Chazot, C. (2002). 'Risk factor analysis for long-term tunneled dialysis catheter-related bacteremias', Nephron; 91: pp. 399-405.

39. Roberts, T., Gregorio, T., Obrador, W., Peter L., Brian S., Pereira J., \& Alan, J. (2004). 'Relationship among catheter insertions, vascular access infections, and anemia management in hemodialysis patients', Kidney International, 66: 2429-2436
40. Hoen, B., Paul-Dauphin, A., Hestin, D., Kessler, M. (1998). EPIBACDIAL: a multicenter prospective study of risk factors for bacteremia in chronic hemodialysis patients. J Am Soc Nephrol; 9(5): 869-876.

41. Hoen, B., Paul-Dauphin, A., Hestin, D., Kessler, M. (1998). EPIBACDIAL: a multicenter prospective study of risk factors for bacteremia in chronic hemodialysis patients. J Am Soc Nephrol; 9(5): 869-876.

42. Powe, N., Jaar, B., Furth. S., Hermann. J., Briggs, W. (1999). 'Septicemia in dialysis patients: incidence, risk factors, and prognosis' Kidney Int; 55(3): pp. 1081-1090.

43. Abdulrahman, S., Al-Mueilo, S., Bokhary, H. Ladipo., G. Al-Rubaish, A. (2002). 'A prospective study of hemodialysis access-related bacterial infections', J Infect Chemother; 8(3): pp. 242-246.

44. Lameire, N., Van Biesen, W. (2010). 'The initiation of renal-replacement therapy--just-intime delivery', N Engl J Med; 363(7): 678-680. 